\title{
HIGHLIGHTS OF THE ISOLDE FACILITY AND THE HIE-ISOLDE PROJECT*
}

\author{
M.J.G. BorGE \\ ISOLDE-PH, CERN, CH-1211 Geneva-23, Switzerland \\ and \\ Instituto de Estructura de la Materia, CSIC \\ Serrano 113bis, 28006 Madrid, Spain
}

(Received December 18, 2015)

\begin{abstract}
The ISOLDE radioactive beam facility is the dedicated CERN installation for the production and acceleration of radioactive nuclei. Exotic nuclei of most chemical elements are available for the study of nuclear structure, nuclear astrophysics, fundamental symmetries and atomic physics, as well as for applications in condensed matter and life sciences. In order to broaden the scientific opportunities beyond the reach of the present facility, the on-going HIE-ISOLDE (High Intensity and Energy) project provides major improvements in energy range, beam intensity and beam quality. A major element of the project is the increase of the final energy of the post-accelerated beams to $10 \mathrm{MeV} / u$ throughout the periodic table. Physics with post-accelerated beams at $4 \mathrm{MeV} / u$ has started this autumn. The increase in energy up to $10 \mathrm{MeV} / u$ is fully funded and it will be implemented at the rate of one cryo-module per year reaching $10 \mathrm{MeV} / u$ for $A / q=4.5$ at the start of 2018. In this contribution, a description of the ISOLDE facility including some highlights will be discussed. The present status of the HIE-ISOLDE project will be described as well as the first experiment realised this year.
\end{abstract}

DOI:10.5506/APhysPolB.47.591

\section{The ISOLDE facility}

The Isotope Separator On-line, ISOLDE, is the radioactive beam facility at CERN, the European Organization for Nuclear Research. It is dedicated to the production, study and research of nuclei far from stability. The facility has garnered unique expertise in radioactive beams over the years since its approval fifty years ago [1]. This ISOL-type facility which started in 1967 at the $600 \mathrm{MeV}$ proton Synchrocyclotron has been located since 1992 at

* Presented at the XXXIV Mazurian Lakes Conference on Physics, Piaski, Poland, September 6-13, 2015. 
the Proton-Synchrotron Booster (PSB) of the CERN accelerator complex. The ISOL method involves in this case the bombardment of a thick target with an intense proton beam of high energy, producing high yields of exotic nuclei with half-lives down to the millisecond range. Pulses of about $3 \times 10^{13}$ protons are sent to the ISOLDE target every 1.2 seconds or multiples of this period resulting in an average beam intensity of $2 p \mu \mathrm{A}[2]$. As many as twenty different solid and liquid target materials are used to produce a wide spectrum of radioactive isotopes covering essentially the whole nuclear chart below uranium. Chemical selectivity is obtained by the right combination of target-ion sources giving rise to a selective production of more than 1300 isotopes of 74 different chemical elements, boron being the last element incorporated this year to this long list.

The ions are subsequently extracted from the ion source, accelerated to $60 \mathrm{keV}$, mass separated according to mass-over-charge ratio using a dipole magnet and sent to the different experimental set-ups. Two mass separator units exist with different mass resolution power, the GPS with $M / \Delta M=$ 1000 and the HRS with $M / \Delta M=5000$. The radioactive beams are used for experiments in the field of nuclear physics ( $\sim 67 \%$ of the beam time), weak interaction studies $(\sim 3 \%)$, solid-state physics studies $(\sim 19 \%)$, nuclear astrophysics $(\sim 4 \%)$, and biology and medicine $(\sim 8 \%)$. The statistics in parenthesis corresponds to year 2014, being a good representation of the annual beam distribution over the different physics domains. Both lowenergy beams up to $60 \mathrm{keV}$ and post-accelerated beams are used in the facility. A general description of the ISOLDE facility is given in [2], with the current layout shown in figure 1.

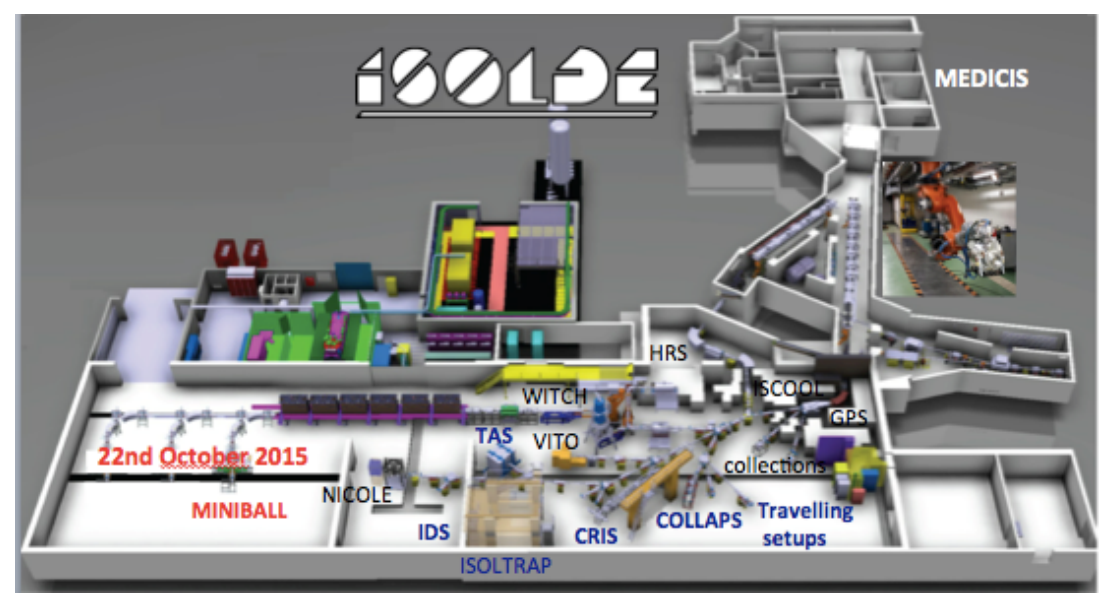

Fig. 1. Layout of the ISOLDE facility. The different parts of the facility and experiments are indicated. The site of the new MEDICIS facility starting in 2016 is also shown. 
The success of ISOLDE is due to the continuous development of new radioactive ion beams and to the improvement of the experimental conditions. Different ways are implemented to purify ISOLDE's beams. The most straightforward way is increasing the wanted isotope over its isobaric contaminants using the high-resolution mass separator [2]. However, using the high-resolution mass separator often induces losses in intensity. Other ways to purify the beam is to make use of the physical and chemical properties of the element. The most elegant and effective way to purify the ion beam is the use of the so-called "Resonant Ionisation Laser Ion Source (RILIS)" [3]. Resonant laser ionisation is an element selective process where atoms are irradiated by two or three lasers exciting the electron in two or three resonant steps into the continuum. RILIS has become extensibly used at ISOLDE [4] because of its ability to efficiently ionize a range of 35 elements with an unmatched degree of element selectivity.

In order to boost the energy of the existing radioactive ion beams of ISOLDE from typically $60 \mathrm{keV}$ to a few $\mathrm{MeV} / u$, a new concept was implemented in which the existing singly-charged ion beams from ISOLDE were accelerated in a universal, fast, efficient and cost-effective way [5]. The concept was based on ion beam cooling and bunching in the buffer gas of a Penning trap [6], charge-state breeding in an Electron Beam Ion Source (EBIS) [7] and post-acceleration in a room-temperature linear accelerator [8] boosting the beam energy to a maximum of about $3 \mathrm{MeV} / u$. The project was called a "Radioactive EXperiment at ISOLDE (REX-ISOLDE)" [5].

The $60 \mathrm{keV}$ radioactive ion beams from ISOLDE are retarded and continuously injected into a Penning trap situated at a high voltage platform. In the trap, the ions are accumulated and, at the same time, cooled via buffer gas collisions. After a fixed time, the ion bunch is extracted from the Penning trap and transferred to the EBIS. The typical duration of the ion bunches extracted from the Penning trap is $10 \mu \mathrm{s}$. After charge breeding to the required mass-to-charge ratio $(A / q)$ between $2.5-4.5$, the ion bunch is extracted from the EBIS, mass separated and injected in the radiofrequency quadrupole (RFQ) accelerator. The room temperature accelerator is typically running with a $10 \%$ duty cycle. The RFQ accelerator is followed by an interdigital H-type (IH) structure with a final energy between 1.1 and $1.2 \mathrm{MeV} / u$, three seven-gap resonators that create an energy range from 0.8 to $2.2 \mathrm{MeV} / u$ and, finally, by a nine-gap resonator boosting the beam energy to a maximum of about $3 \mathrm{MeV} / u$.

While the original goals of the REX-ISOLDE project were limited to energies up to $2 \mathrm{MeV} / u$ and masses below $A=50$, the universal concept proved to be very successful and meanwhile beams with $2<A / q \leq 4.5$ and with masses up to 224 have been accelerated up to $3 \mathrm{MeV} / u$, with efficiencies varying from $\sim 2$ to $20 \%$. The experimental program with post-accelerated 
beams focussed on Coulomb excitation, few-nucleon transfer reaction studies of light nuclei. To learn more about REX-ISOLDE, the experimental devices used and the scientific achievements, see [9].

\subsection{ISOLDE highlights}

ISOLDE has pioneered many achievements both at the level of designing new devices and of producing frontier physics. In the following, some of the recent highlights will be presented.

\subsubsection{Exotic decay studies at ISOLDE}

Close to the beta-stability line, all beta-decays will populate particle bound states, i.e. states that are long lived and, therefore, have narrow widths, less than $1 \mathrm{keV}$. Moving towards the drip-lines, a larger and larger fraction of $\beta$-decays will feed states that are unbound for the proton emission in the neutron-deficient side and neutron emission in the neutron-rich side or alpha emission that is possible in both sides of the valley of stability. To the point that close to the drip-line beta-delayed particle emission often becomes the dominating decay mode. A general overview of the different decay modes and their impact on the knowledge on nuclear structure near the drip-line and beyond can be found in recent reviews $[10,11]$.

I. ${ }^{6} \mathrm{He} \beta$-decay into the $\alpha+d$ continuum, revisited.

The rare decay mode of ${ }^{6} \mathrm{He}$ into the $d+\alpha$ continuum was revisited recently at ISOLDE using an optical time projection chamber (OTPC). The ${ }^{6} \mathrm{He} \beta$-decay mainly proceeds to the ${ }^{6} \mathrm{Li}$ ground state and with a probability of the order of $10^{-6}$ breaks into a deuteron and an alpha. This decay has the peculiarity to proceed directly to the continuum. It is considered to represent a peripheral decay of the two neutrons of the halo into deuteron in the outskirts of the ${ }^{6} \mathrm{He}$ nucleus. The branching ratio and the shape of the energy spectrum are very sensitive to details of the initial state and to the interaction between the deuteron and daughter final state. Previous experiments suffered from huge beta background from the main branch limiting the sensitivity of the deuteron spectrum, to energy above $350 \mathrm{keV}$. To be able to go lower in energy in the deuteron spectrum, a new technique was applied using an OTPC which allows to reconstruct the tracks for the deuteron and alphas being insensitive to the beta electrons. This was a challenge for ISOLDE as we have never post-accelerated He-beam due to the cooling gas in the REX-TRAP. Thanks to the large production of ${ }^{6} \mathrm{He}$ beam at ISOLDE even with a reduced efficiency intense bunches of post-accelerated ${ }^{6} \mathrm{He}$ ions were implanted into the OTPC, where the decays with emission of charged particles were recorded. Figure 2 shows on the 
left-hand side one of the events recorded by the CCD (upper photo) and in the PMT, bottom spectrum. This novel technique allowed to extend the low-energy end of the spectrum down to a deuteron energy of $100 \mathrm{keV}$, see on the right-hand side of figure 2 the $E_{d \alpha}$ spectrum compared with the one of Raabe et al. [12] used for normalisation. The new branching ratio for this process amounts to $2.78(18) \times 10^{-6}$ similar to previous values but much more precise, see [12] and references therein. The shape of the spectrum is found to be in a good agreement with a three-body model [13], while the total intensity is about $20 \%$ larger than the predicted one. For more details of this beautiful experiment, see [14].

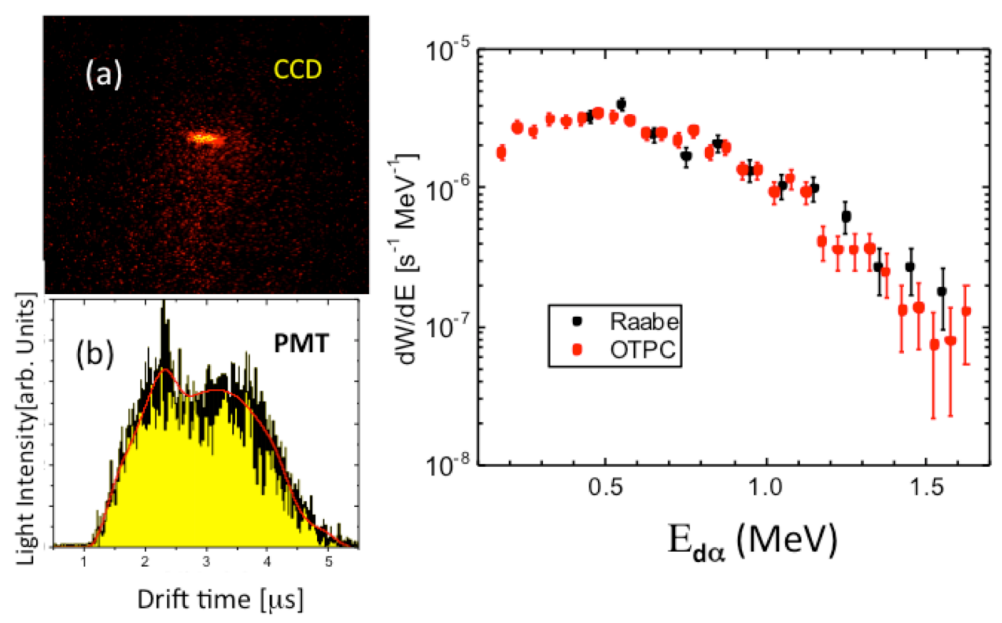

Fig. 2. On the left-hand side, (a) shows one of the $\alpha+d$ events recorded in the CCD camera with the corresponding PMT signal shown in (b). On the right-hand side, the transition probability of the $\alpha+d$ branch in the $\beta$-decay of ${ }^{6} \mathrm{He}$ versus the center-of-mass energy, $E_{d \alpha}$. The new data is compared with the one of Raabe $e t$ al. [12] used for normalisation. Notice the large sensitivity improvement of the new data at low energies.

II. First determination of beta-delayed proton branch in the neutron-rich halo nucleus ${ }^{11} \mathrm{Be}$.

The halo structure not only can be responsible for the existence of $\beta$-delayed deuteron emission to the continuum but could allow a very exotic decay mode consisting of the emission of a proton from an $n$-rich nucleus. $\beta^{-}$-decay and proton emission take a nucleus in opposite directions on the nuclear chart so $\beta^{-}$-delayed proton emission, $\beta^{-} p$, is available in a few nuclei due to the limited energy window, $Q_{\beta^{-} p}=782 \mathrm{keV}-S_{n}, S_{n}$ being the neutron separation energy. This $\beta^{-} p$ decay mode is expected in $1 n$-halo nuclei due to both energetics and the more pronounced single-particle behaviour of 
haloes. The low-decay energy implies that the branching ratio for this decay mode is very low and it is estimated for the most favourable case, ${ }^{11} \mathrm{Be}$, to be around $10^{-8}$. This rare decay mode has been recently observed at ISOLDE in the decay of ${ }^{11} \mathrm{Be}$. To investigate the $\beta^{-} p$ in ${ }^{11} \mathrm{Be}$, one has to face the difficulty of detecting protons of maximum energy of $280.7(3) \mathrm{keV}$ with a very low branching ratio. Therefore, the idea is to produce a very intense and pure sample, and search for the $\beta^{-} p$ daughter with a level of sensitivity better than $10^{-8}$. Considering that the half-lives of ${ }^{11} \mathrm{Be}$ and the $\beta^{-} p$ daughter, ${ }^{10} \mathrm{Be}$, are very different, $13.8 \mathrm{~s}$ and $1.5 \times 10^{6}$ years, respectively; one can consider to make a sample and send it to a state-of-art accelerator mass spectrometry (AMS) device that has the required sensitivity.

In a first attempt, the signal was not strong enough and a $\beta^{-} p$ branching ratio of $2.5(25) \times 10^{-6}$ was obtained [15]. Due to the improvements in the production of ${ }^{11} \mathrm{Be}$ at ISOLDE and a higher sensitivity in the AMS detection of ${ }^{10} \mathrm{Be}$, the experiment was tried again in 2012. Samples of ${ }^{11} \mathrm{Be}$ and the possible contaminant ${ }^{11} \mathrm{Li}$ were collected. The produced activity was monitored by the measurements of the gamma activity connected to the respective decays. The ${ }^{10} \mathrm{Be}$ AMS measurements were performed at the Vienna Enviromental Research accelerator (VERA). The branching ratio of the $\beta^{-} p$ decay mode in ${ }^{11} \mathrm{Be}$ was determined by detecting the number of final nucleus ${ }^{10} \mathrm{Be}$ obtained by the AMS method with respect to the deduced number of ${ }^{11} \mathrm{Be}$ ions collected in the original sample. The new branching ratio value is of $8.3(9) \times 10^{-6}$. This branching ratio is astonishingly large and consistent with the previous measurement [15]. No existing calculations reproduce the magnitude. This unexpected high $\beta$-decay branch can only be understood if one assumes that the decay proceeds into an unobserved single proton resonance in ${ }^{11} \mathrm{~B}$. For more details, see [16].

III. Beta-delayed particle emission from ${ }^{20-21} \mathrm{Mg}$ : a new decay mode identified in ${ }^{21} \mathrm{Mg}$.

The beta-decay of the drip-line nucleus ${ }^{20} \mathrm{Mg}$ gives important information on resonances in ${ }^{20} \mathrm{Na}$, which are relevant for the astrophysical rp-process. In particular, the spin and parity of the $2645 \mathrm{keV}$ level in ${ }^{20} \mathrm{Na}$ is unsettled. This resonance is situated in the Gamow-window of the ${ }^{19} \mathrm{Ne}(p, \gamma)^{20} \mathrm{Na}$ reaction from the breakout sequence of the CNO cycle. Thus, it is essential to understand the reaction rate.

Previous studies have been done at in-flight facilities. The ability of producing ${ }^{20} \mathrm{Mg}$ at ISOLDE gives new possibilities. First, the purity of the beam at ISOL facilities allows a much cleaner spectrum of the decay to be measured. Secondly, the lower energy of the ion beam allows for implantation in a thin window of e.g. a Gas-Si telescope. The $\beta$-decay study of ${ }^{20} \mathrm{Mg}$ was completed recently with the new decay station, IDS, described below. 
The ${ }^{21} \mathrm{Mg}$ beam was studied for proton energy calibration purpose. The advantage of using a Gas-Si telescope is the possibility to allow for a direct distinction of the various decay branches. The beam was implanted in the polypropylene window of the gas detector. Two Si-detectors of $300 \mu \mathrm{m}$ and $500 \mu \mathrm{m}$ each where placed behind the gas detector and embedded in the same $\mathrm{CF}_{4}$ gas. Opposite to the Gas-Si-Si telescope is a $61 \mu \mathrm{m}$ thick $5 \times 5 \mathrm{~cm}^{2}$ DSSSD backed by a $1000 \mu \mathrm{m}$ thick Si pad detector. The very compact setup used in this experiment and shown in figure 3 has allowed to identify a new decay branch, $\beta p \alpha$. The $\beta p$ spectrum has allowed to identify 27 decay branches, seven of which are new, including a high-energy proton branch of $7.2 \mathrm{MeV}$. By studying the line shape of the proton peaks and including possible interferences the widths, spins and parities of the resonances of ${ }^{21} \mathrm{Na}$ are obtained. The new results give good agreement with previous inelastic proton reaction studies [17]. Five $\alpha$ branches can be distinguished in the two-dimension spectrum on the right-hand side of figure 3 with centre-ofmass energies between 0.9 and $3.1 \mathrm{MeV}$ accounting for a total branching of $1.32(4) \times 10^{-3}$. The very low $\alpha$ peak with laboratory energy of $714(12) \mathrm{keV}$ coincided in energy with a known $\alpha$ particle transition from ${ }^{20} \mathrm{Ne}$ to ${ }^{16} \mathrm{O}$. Strong support to this assignment comes from the observation in the DSSSD detector of a proton peak in coincidence with this $\alpha$ peak. In this way, one can establish a new decay mode in ${ }^{21} \mathrm{Mg}$ with a branch of $1.6(3) \times 10^{-4}$ from the isobaric analog state in ${ }^{21} \mathrm{Na}$ to the $8.054 \mathrm{MeV}$ state in ${ }^{20} \mathrm{Ne}$ with subsequent $\alpha$ emission to the ground state of ${ }^{16} \mathrm{O}$ [18]. The $\beta p \alpha$ decay mode is very rare and it has only been observed previously in ${ }^{9} \mathrm{C}$ and ${ }^{17} \mathrm{Ne}$. To complete the series, this decay mode should be observed in ${ }^{13} \mathrm{O}$ and ${ }^{23} \mathrm{Si}$.
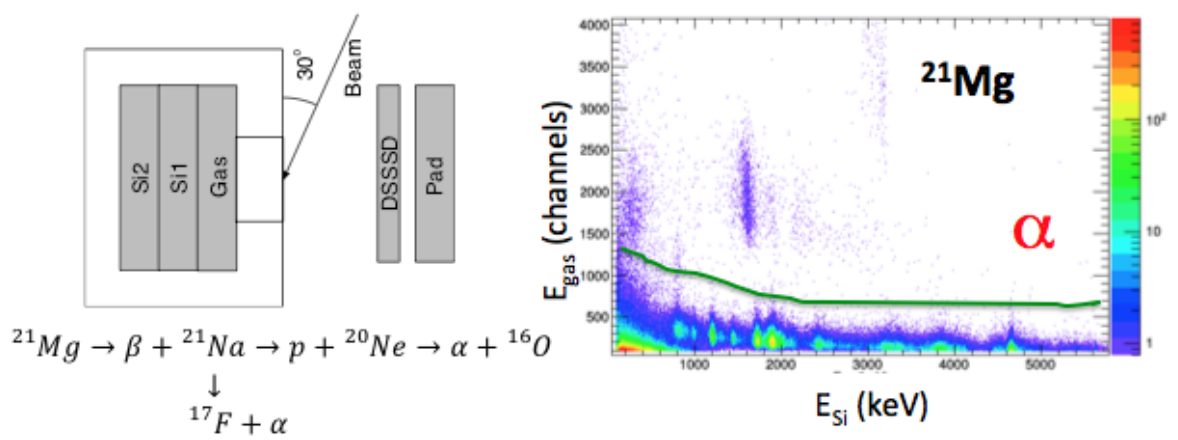

Fig. 3. On the left-hand side, a drawing of the set-up used to study the ${ }^{20-21} \mathrm{Mg}$ decay is shown. The beam is implanted in the polypropylene window of the gas detector of a Gas-Si-Si telescope, opposite to it there is another DSSSD-Si telescope for coincidence study. On the right-hand side, the two dimension $\Delta E-E$ plot using the Gas-Si telescope of the $\beta$-delayed charged particles of ${ }^{21} \mathrm{Mg}$. Very clearly we can distinguish alpha and proton branches. 


\subsubsection{First experiments with the new fixed ISOLDE Decay Station, IDS}

During the CERN long shut down, we took the opportunity to put together a permanent set-up for decay studies. The basic parts are an array consisting of four clover type HPGe detectors in close geometry and ancillary detectors surrounding an implantation chamber with possibility of a tape station and a large plastic scintillator for beta detection. Among the ancillary detectors, we count with a 12-fold Miniball type triple HPGe detector to enhance the $\gamma-\gamma$ coincidences studies. Three $\mathrm{LaBr}_{3}$ detectors can be coupled to the system for fast time half-lives measurements of excited states. The system has been also coupled to the neutron Time-of-Flight array VANDLE (Versatile Array for Neutron Detection at Low Energies) this year with great success. Both last year and this year, very exotic neutron-deficient nuclei such as ${ }^{31} \mathrm{Ar}$ and ${ }^{20} \mathrm{Mg}$ were measured by detection of charged particles and $\gamma$-rays by the using of the MAGISOL Plug-in chamber that contains an array of 5 DSSSD backed by unsegmented Si-pad detectors in $\Delta E-E$ telescope configuration, to learn more, see [19]. Geant4 simulation has been done to obtain the efficiencies of the different configurations of this highly performing very versatile set-up.

In these two years, several physics cases have been addressed. For instance, the high-efficiency beta-gamma set-up was used for the study of the intruder configurations in ${ }^{34} \mathrm{Al}$ and ${ }^{34} \mathrm{Si}$ from the beta-decay of ${ }^{34} \mathrm{Mg}$. These measurements were followed by ISOLTRAP measurements of the mass of the ground state and isomeric state in ${ }^{34} \mathrm{Al}$ in dispute since long time. During the first successful fast-timing measurement at the ISOLDE Decay Station (IDS), we investigated the properties of low-lying excited levels in ${ }^{129} \mathrm{Sn}$. This nucleus has three neutron holes compared to the doubly magic ${ }^{132} \mathrm{Sn}$. It represents a good case for testing shell-model calculations near the $N=82$ shell closure due to the reduced degrees of freedom for low excitation states. The measured half-live of the $1 / 2^{+} 315.3 \mathrm{keV}$ level in ${ }^{129} \mathrm{Sn}$ is by a factor of 100 faster than expected using the CD-Bonn effective interaction. A renormalisation of the M1 effective interaction is needed to conceal the experimental result and the theoretical expectations [20]. The same set-up has been applied to the study of shape coexistence and presence of static octupole deformation in ${ }^{148-152} \mathrm{Ba}$ and to the characterisation of the lowlying $0^{+}$and $2^{+}$states of ${ }^{68} \mathrm{Ni}$ by measuring lifetimes using the fast-timing

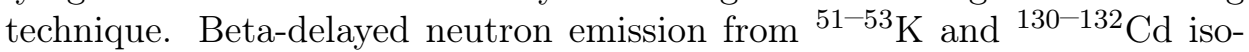
topes using VANDLE from Oak Ridge has been realised this summer. The $N=50,82$ regions are of special interest for beta-decay measurements as the decay is complicated by the fact that valence neutrons and protons occupy different major harmonic shells. Under these conditions, the normally inert nucleons in the core are allowed to play a role due to the small overlap of the valence neutrons with low-energy states in the daughter. The large 
energy gap at $N=50$ implies the allowed decay of core neutrons occurs to neutron unbound states. To investigate the link between large neutron emission probabilities and core breaking, we measured the beta-delayed neutron emission of $n$-rich Cd isotopes. In the case of ${ }^{132} \mathrm{Cd}$, beta decay is expected to populate quasi-single particle states thanks to the small valence space in ${ }^{132} \operatorname{In},\left(Z_{v}=-1, N_{v}=-1\right)$, and be dominated by the beta transition of the $g_{7 / 2} \rightarrow g_{9 / 2}$. This allowed us to have a unique insight to the nuclear structure of states making ${ }^{132} \mathrm{Sn}$.

The pure beam of ${ }^{132} \mathrm{Cd}$ ions was implanted in the Leuven tape system, which moved the activity away after $600 \mathrm{~ms}$. The experimental set-up consisted of 2 large plastic scintillators for beta detection with $90 \%$ efficiency, see figure 4, 4 HPGe clover detectors, $4 \%$ at $1 \mathrm{MeV}$, and 26 detector bars from the VANDLE array, $6 \%$ efficiency at $1 \mathrm{MeV}$. The gamma spectrum following beta decay of ${ }^{132} \mathrm{Cd}$ is shown in figure 5 , with lines identified from the decay of both ${ }^{131} \mathrm{In}$ isomers. The $\gamma$-lines at $988 \mathrm{keV}$ and at $1221 \mathrm{keV}$ in ${ }^{131} \mathrm{In}$ [21] are clearly identified, indicating they are populated following neutron

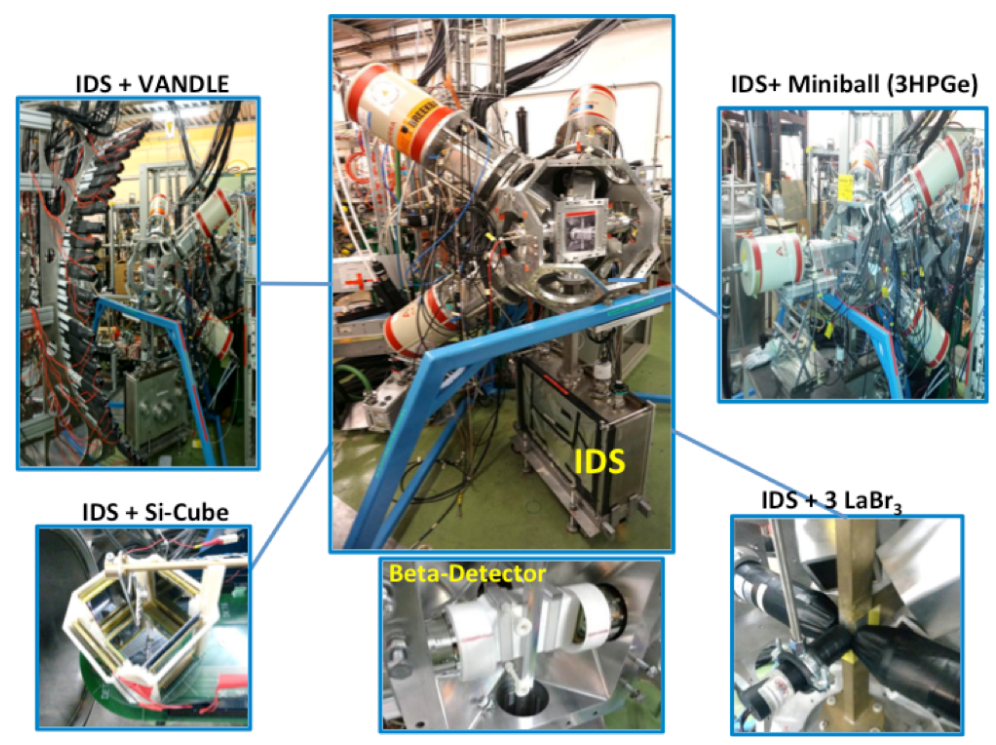

Fig. 4. The versatile new permanent decay station, IDS, consist of 4 clover HPGe detectors, a tape station and a beta detector. The photos around show clockwise the ancillary elements: the fifth HPGe, a Miniball triple HPGe cluster, the fast timing set-up with $3 \mathrm{LaBr}_{3}$, the new beta detector surrounding the tape, the DSSSD-Si telescopes for charged particle detection and the time-of-flight neutron detector, VANDLE. In this way, studies of exotic nuclei from the very neutron deficient to the very neutron rich can be realised. 


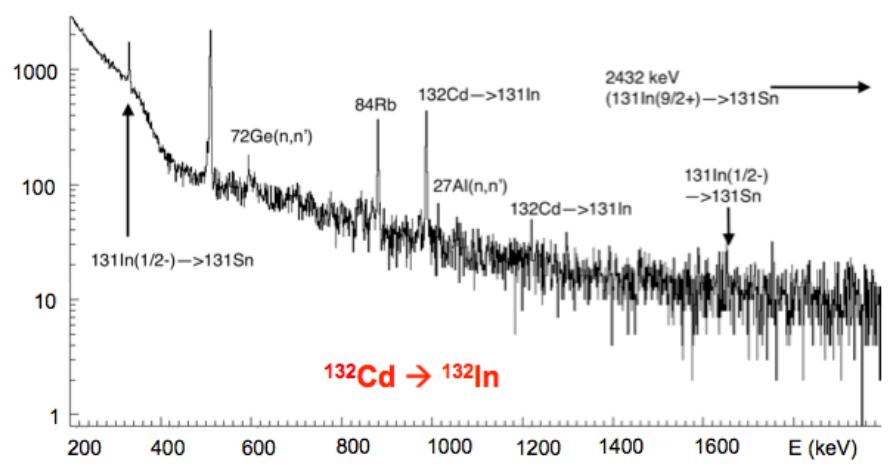

Fig. 5. Beta gated gamma spectrum following the decay of ${ }^{132} \mathrm{Cd}$. All major transitions are marked (except for the $511 \mathrm{keV}$ peak). Two lines at 988 and $1221 \mathrm{keV}$ previously observed in the decay of ${ }^{131} \mathrm{Cd}[21]$ are identified, indicating they are populated following neutron emission. Intriguingly, no transitions in or from the decay of ${ }^{132}$ In are observed, indicating the neutron branching ratio must be close to $100 \%$.

emission. Interestingly, no gamma lines are observed that can be assigned from states in or even from the beta-decay of ${ }^{132} \mathrm{In}$. This indicates that the neutron branching ratio must be close to $100 \%$. The neutron spectrum is currently being processed. Preliminary analysis shows an intense neutron emission $1.5 \mathrm{MeV}$ above the neutron separation energy. Calculations of the Gamow-Teller decay strength using Random Phase Approximation to calculate levels in ${ }^{132}$ In predict the largest transition strength to correspond to the $\nu g_{7 / 2}^{-1}-\pi g_{9 / 2}^{-1}$ configuration. The discrepancy between the observed and calculated energies will give us an insight in the structure of the ${ }^{132} \mathrm{Sn}$ core.

\subsubsection{Study of mean square radius of $n$-deficient heavy nuclei}

In recent years, the RILIS team in collaboration with several experimental groups at ISOLDE have performed extensive laser and nuclearspectroscopic studies of shape evolution/coexistence in the lead region using the technique of in-source laser spectroscopy [22]. This is the most sensitive laser spectroscopy method available at ISOL facilities and has enabled the measurement of isotopes with a yield as low as 0.1 ions/second. This year, At $(Z=85), \mathrm{Au}(Z=79)$ and $\mathrm{Hg}(Z=80)$ have been studied employing a combination of particle detection techniques: $(\alpha / \beta / \gamma)$ with the Windmill set-up from Leuven (Belgium); direct ion counting with the ISOLTRAP multi-reflection time-of-flight mass spectrometer (MR-TOF MS) [23]; and ion beam current measurements using the ISOLDE Faraday cups. The multi-reflection time-of-flight spectrometer with a resolution of $M / \Delta m=$ 
$10^{6}$ was essential to study the isotopes of gold and astatine. The combination of all these methods has allowed to determine the hyperfine structure measured by RILIS of the neutron deficient $\mathrm{Hg}$ isotopes from $A=177$ to 181 . The mean square radii show absence of the odd-even staggering observed in the ${ }^{181-184} \mathrm{Hg}$ isotopes. This confirms the predictions that well below the mid-shell neutron number $N=104$, the structure of the Hg-isotopes becomes spherical [24]. Using the same techniques, the mean square radii of the $n$-deficient $\mathrm{Au}$ isotopes from $A=176-183$ were determined. The results show that the deformed $\mathrm{Au}$ isotopes are symmetric around the mid-shell $N=104$ and return to sphericity for neutron deficient isotopes lighter than ${ }^{179} \mathrm{Au}$.

\section{The HIE-ISOLDE project}

The HIE-ISOLDE project (HIE stands for High Intensity and Energy), intends to improve the experimental capabilities at ISOLDE over a wide front [25]. The main features are to boost the energy of the beams, going in steps from previous $3 \mathrm{MeV} / u$ via $5.5 \mathrm{MeV} / u$ to finally $10 \mathrm{MeV} / u$. An increase of roughly sixfold in the production is expected due to an increase in intensity and energy of the proton injector. In addition, improvements in several aspects of the secondary beam properties such as purity, ionisation efficiency and optical quality are addressed in the project. Major project components include a new superconducting (SC) linear accelerator (LINAC). The SC LINAC is based on a quarter-wave resonator (QWR) geometry with twenty high- $\beta$ cavities cooled by helium and installed in four cryo-modules to reach the beam energy of $10 \mathrm{MeV} / u$ for $A / q=4.5$. Each cryo-module contains five cavities based on $101.28 \mathrm{MHz}$ niobium-sputtered copper $(\mathrm{Nb} / \mathrm{Cu})$ QWR and one solenoid. Due to the limited space, it has been necessary to design and build accelerating cavities with a very high voltage gradient of $6 \mathrm{MV} / \mathrm{m}$ and low heat dissipation below $10 \mathrm{~W}$. The transverse focusing is achieved using four superconducting solenoids housed inside the cryo-modules maximising the transverse acceptance. Figure 6 displays the full post-accelerated beam facility at ISOLDE. At the exit of the LINAC, three identical beam lines at $90^{\circ}$ are planned and two of them are fully operative to allocate the different experiments. The approved physics cases expand over the wide range of post-accelerated beams available at ISOLDE. The increase in energy of the radioactive beams will enhance the cross section in most of the cases and the accessibility to detailed nuclear structure information at higher excitation energy. The proposed studies will be realised with the existing workhorses Miniball [26] $\gamma$ array for Coulex together with T-REX [27] for transfer reaction studies in the first beam line. New instrumentation for transfer reaction studies such as the active 


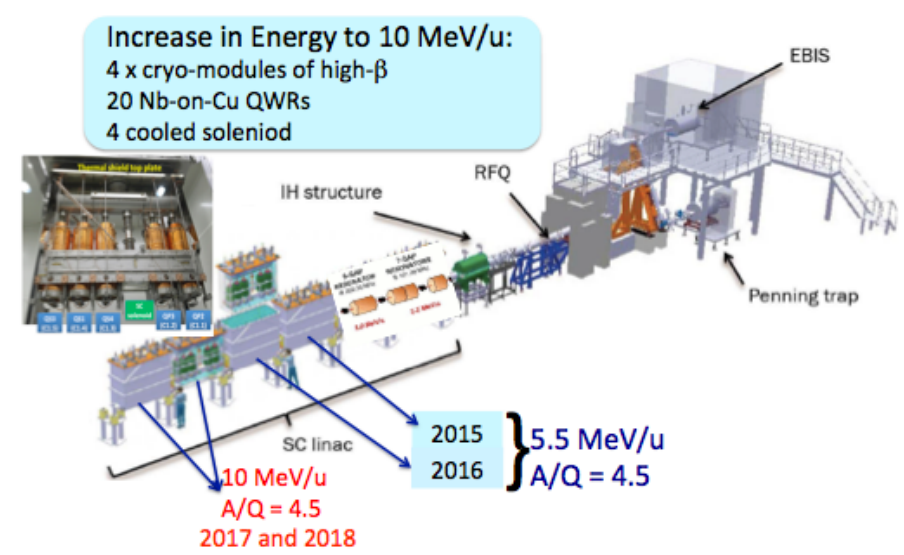

Fig. 6. The 3D-plot shows the full post accelerated beam facility at ISOLDE. The ion beam is cooled and bunched in the buffer gas of the REX-trap [6], charge-state breed in an Electron Beam Ion Source (EBIS) [7], post-accelerated in a roomtemperature linear accelerator [8] and injected in the new superconducting, SC, LINAC. A photo with the 5 cavities inside the cryo-module is shown. The time scale for the implementation of the different cryo-modules is indicated.

targets MAYA [28] and the future ACTAR, a new general purpose scattering chamber, the two arms CORSET set-up from GSI, etc. will be placed in the second beam line. The Miniball array will be complemented with an electron spectrometer, SPEDE, to realise Coulomb excitation studies of odd-mass heavy nuclei. While writing these pages, the day-one experiments are taking data. We have started with the study of the evolution of the nuclear structure along the $n$-rich zinc isotopes close to $N=50$. This study will probe recent shell-model calculations in this area of the nuclear chart. Excitation energies and connecting $B(\mathrm{E} 2)$ values are deduced from the ejectile particle measurements in the CD (see below) and the $\gamma$-ray measurements in Miniball through a multiple Coulomb excitation experiment with laser ionised beams of ${ }^{74-76} \mathrm{Zn}$. Coulomb excitation at low beam energy offers an excellent tool to obtain energies of the low-lying levels, including non-yrast states, as well as $B(\mathrm{E} 2)$ values. Miniball [26] $\gamma$ array consists of eight cryostats, each containing three individual germanium crystals, that are mounted in close geometry around the target position. For experiments in very close geometry like this one, the solid-angle coverage is of $\sim 60 \%$ of $4 \pi$ and photo-peak efficiency for $1.3 \mathrm{MeV} \gamma$-ray of about $8 \%$. The spherical Miniball detection chamber is equipped with a double sided silicon strip detector (DSSSD) of the CD type [29]. The DSSSD covers an angular range typically from $16^{\circ}$ to $53^{\circ}$ laboratory angle in the forward direction. 
On October $22^{\text {nd }}, 2015$ in the afternoon, the first radioactive beam of ${ }^{74} \mathrm{Zn}$ was observed in the $\mathrm{CD}$ detector of the Miniball chamber. Figure 7 shows a comparison of the two Coulex spectra obtained for the postaccelerated ${ }^{74} \mathrm{Zn}$ beam on a Pt thin target at the present energy of $4 \mathrm{MeV} / u$ and the previous one of $2.87 \mathrm{MeV} / u$. In previous Coulex measurements of ${ }^{74-76} \mathrm{Zn}$, the level $4^{+}$was barely populated [30]. The poor statistics collected in the $4^{+} \rightarrow 2^{+}$transition gave a quite large error bar in the extracted transitional matrix element. With the present new data, we will get a much better determination of the $B\left(\mathrm{E} 2 ; 4^{+} \rightarrow 2^{+}\right) / B\left(\mathrm{E} 2 ; 2^{+} \rightarrow 0^{+}\right)$ratio allowing for a model-independent determination of the collective character of these nuclei and their progressive reduction of collectivity when approaching the magic number $N=50$ at ${ }^{80} \mathrm{Zn}$. Further, the discrepancy between $B\left(\mathrm{E} 2 ; 4^{+} \rightarrow 2^{+}\right)$ values for ${ }^{74} \mathrm{Zn}$ obtained previously at ISOLDE [30] and at Legnaro [31] will be removed.

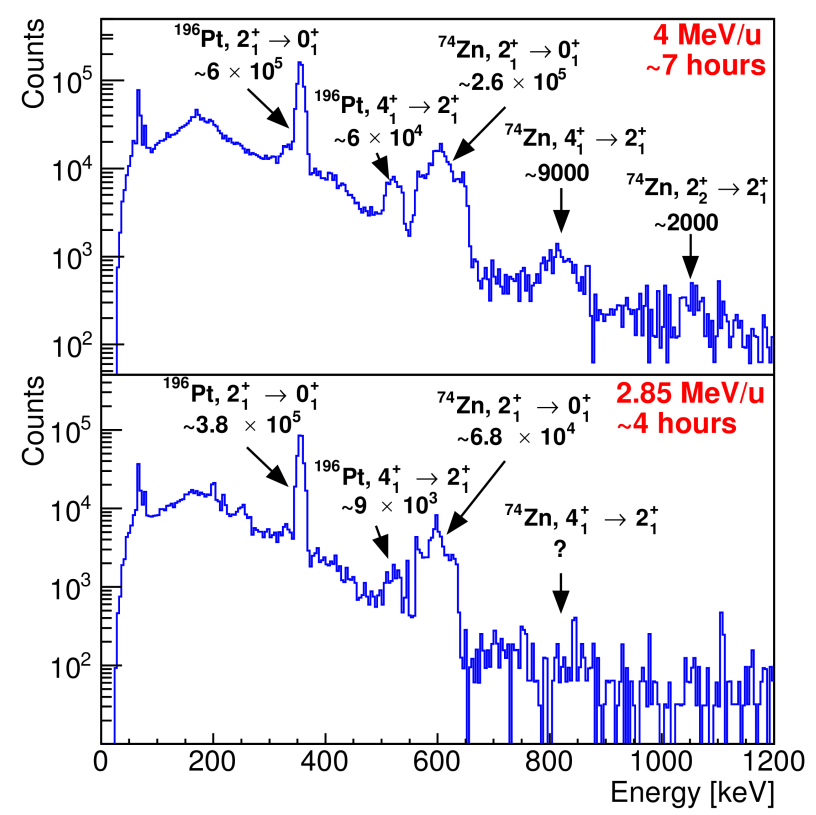

Fig. 7. The figure illustrates the Coulomb excitation $\gamma$ spectra of a laser ionised ${ }^{74} \mathrm{Zn}$ beam on a $\mathrm{Pt}$ target obtained in prompt coincidence with a recoiling ejectile. Shown is the spectrum obtained this year at $4 \mathrm{MeV} / u$ (upper part) and at the previous REX energies of $2.87 \mathrm{MeV} / u$ (bottom part). The increase in beam energy enlarges the physics window available. 


\section{Summary and outlook}

The future of ISOLDE is bright. The knowledge accumulated over decades on how to construct targets and ion sources tailored to release pure beams of specific elements are one of ISOLDE strong points. With more than 50 years since approval, ISOLDE remains as the pioneer ISOL-installation both at the level of designing new devices and production of frontier physics. The first phase of HIE-ISOLDE has started for physics with first radioactive beam on the $22^{\text {nd }}$ of October 2015. The first experiment explored the evolution of nuclear structure near ${ }^{78} \mathrm{Ni}$ by performing multi-step Coulomb excitation in $n$-rich $\mathrm{Zn}$ isotopes. We expect to complete the increase of energy of post accelerated beams at the rate of one cryo-module per year up to $10 \mathrm{MeV} / u$ for $A / q=4.5$ at the start of 2018 .

\section{REFERENCES}

[1] K. Blaum, M.J.G. Borge, B. Jonson, P. Van Duppen, in: Advanced Series on Direction in High Energy Physics, Vol. 23 (2015), 60 Years of CERN

Experiments and Discoveries, Eds. H. Schopper, L. Di Lella, p. 415.

[2] E. Kugler, Hyperfine Interact. 129, 23 (2000).

[3] V.I. Mishin et al., Nucl. Instrum. Methods B 73, 550 (1993).

[4] V.N. Fedoseyev et al., Hyperfine Interact. 129, 409 (2000).

[5] D. Habs et al., Hyperfine Interact. 129, 43 (2000).

[6] F. Ames et al., Nucl. Instrum. Methods A 538, 17 (2005).

[7] B. Wolf et al., Rev. Sci. Instrum. 73, 682 (2002).

[8] T. Siebert et al., Nucl. Phys. A 701, 656 (2002).

[9] P. Van Duppen, K. Riisager, J. Phys. G: Nucl. Part. Phys. 38, 024005 (2011).

[10] B. Blank, M.J.G. Borge, Prog. Part. Nucl. Phys. 60, 403 (2008).

[11] M. Pfützner, M. Karny, L.V. Grigorenko, K. Riisager, Rev. Mod. Phys. 84, 567 (2012).

[12] R. Raabe et al., Phys. Rev. C 80, 054307 (2009).

[13] E.M. Tursunov, D. Baye, P. Descouvemont, Phys. Rev. C 73, 014303 (2006); [Erratum ibid. 74, 069904 (2006)].

[14] M. Pfützner et al., Phys. Rev. C 92, 014316 (2015).

[15] M.J.G. Borge et al., J. Phys. G 40, 035109 (2013).

[16] K. Riisager et al., Phys. Lett. B 732, 305 (2014).

[17] M.V. Lund et al., Eur. Phys. J. A 51, 113 (2015).

[18] M.V. Lund et al., Phys. Lett. B 750, 356 (2015).

[19] I. Marroquin et al., Acta Phys. Pol. B 47, 747 (2016), this issue.

[20] R. Lica et al., in preparation. 
[21] Z.Y. Xu et al., Phys. Rev. Lett. 113, 032505 (2014); G. Lorusso et al., Phys. Rev. Lett. 114, 192501 (2015).

[22] B.A. Marsh et al., Nucl. Instrum. Methods B 317, 550 (2013).

[23] R.N. Wolf et al., Int. J. Mass. Spectrom. 349-350, 123 (2013);

F. Wienholtz et al., Mass Measurements and Laser Spectroscopy Using ISOLTRAP's Multi-reflection Time-of-Flight Device, presented at the XXXIV Mazurian Lakes Conference on Physics, Piaski, Poland, September $6-13,2015$.

[24] B.A. Marsh et al., in preparation.

[25] HIE-ISOLDE, the Scientific Opportunities, Eds. K. Riisager, P. Butler, M. Huyse, R. Krücken, CERN Report, CERN-2007-008.

[26] J. Eberth et al., Progr. Part. Nucl. Phys. 46, 389 (2001).

[27] V. Bildstein et al., Eur. Phys. J. A 48, 85 (2012).

[28] C.E. Demonchy et al., Nucl. Instrum. Methods A 583, 341 (2007).

[29] A. Ostrowski et al., Nucl. Instrum. Methods A 480, 448 (2002).

[30] J. Van de Walle et al., Phys. Rev. C 79, 014309 (2009).

[31] C. Louchart et al., Phys. Rev. C 87, 054302 (2013). 